\title{
ON COMPACT EINSTEIN-KAEHLER MANIFOLDS
}

\author{
BANG-YEN CHEN ${ }^{1}$ AND KOICHI OGIUE ${ }^{2}$
}

ABSTRACT. A characterization of a complex space form among compact Einstein-Kaehler manifolds is given in terms of Chern classes.

1. Introduction. Let $M$ be a Kaehler manifold and $c_{k}$ the $k$ th Chern class of M. A Kaehler manifold of constant holomorphic sectional curvature is call a complex space form. If $M$ is an $n$-dimensional complex space form $(n \geq 2)$, then $c_{2}=n c_{1}^{2} / 2(n+1)$ (cf. [1]). In [1] we considered the converse.

The purpose of this paper is to study similar problems under somewhat weaker assumption.

Theorem. Let $M$ be an $n$-dimensional compact Kaehler manifold $(n \geq 2)$. If

(i) $c_{1}^{n-2} c_{2}=n c_{1}^{n} / 2(n+1)$, and

(ii) $M$ is Einstein, then $M$ is either a complex space form or a Kaehler manifold with vanishing Ricci tensor.

2. Preliminaries. Let $\|$ be an $n$-dimensional Kaehler manifold. Let (1) $1, \ldots,(1)^{n}$ be a local field of unitary frames. Then the Kaehler metric is written as $g=1 / 2 \Sigma\left(\omega^{\alpha} \otimes \bar{\omega}^{\alpha}+\bar{\omega}^{\alpha} \otimes \omega^{\alpha}\right)$ and the fundamental 2-form is given by $\Phi=1 / 2 \sqrt{-1} \Sigma \omega^{a} \wedge \bar{\omega}^{a}$. Let $\Omega_{\beta}^{a}=\Sigma R_{\beta \gamma}^{a} \delta^{\gamma} \wedge \bar{\omega}^{\delta}$ be the curvature form of $M$. Then the curvature tensor of $M$ is the tensor field with local components $R_{B \gamma \bar{\delta}}^{a}$, which will be denoted by $R$. The Riccitensor $S$ and the scalar curvature $\rho$ are given by

$$
S=\frac{1}{2} \sum\left(R_{\alpha \bar{\beta}^{\beta}} \omega^{\alpha} \otimes \bar{\omega}^{\beta}+\bar{R}_{c \bar{\beta}^{\bar{\omega}}} \overline{-}^{\alpha} \otimes \omega^{\beta}\right), \quad \rho=2 \sum R_{\alpha \bar{a}},
$$

where $R_{a \bar{\beta}}=2 \sum R_{a \gamma \bar{\beta}}^{\gamma}$. We denote by $\|R\|$ and $\|S\|$ the length of the curvature tensor and the Ricci tensor, respectively, so that $\|R\|^{2}=16 \Sigma R_{\beta y}^{a} \bar{\delta} R_{a \delta \bar{\gamma}}^{\beta}$ and $\|S\|^{2}=2 \sum R_{a \bar{\beta}} R_{\beta \bar{\alpha}}$.

We prepare the following general result.

Lemma. Let $M$ be an n-dimensional Kaehler manifold. Then

Received by the editors August 16, 1974 and, in revised form, October 25, 1974.

AMS (MOS) subject classifications (1970). Primary 53C55; Secondary 57D20.

Key words and phrases. Compact Einstein-Kaehler manifold, complex space form, Chern class.

1 Work done under partial support by NSF Grant GP-36684.

2 Work done under partial support by NSF Grant GP-36684 and the Matsunaga Science Foundation. 


$$
1 / 2 n(n+1)\|R\|^{2} \geq 2 n\|S\|^{2} \geq \rho^{2} .
$$

The first equality holds if and only if $M$ is a complex space form, and the second equality holds if and only if $M$ is Einstein.

Proof. The first inequality is obtained by considering the length of the tensor field with local components $R_{\beta \gamma \bar{\delta}}^{a}-[2(n+1)]^{-1}\left(\delta_{\gamma}^{\alpha} R_{\beta \bar{\delta}}+\delta_{\beta}^{\alpha} R_{\gamma} \bar{\delta}\right)$. It is well known that this tensor field vanishes if and only if $M$ is a complex space form (cf., for example, [2]).

The second inequality is obtained by considering the tensor field with local components $R_{a \bar{\beta}}-\rho \delta_{a \beta^{\prime}} / 2 n$. It is clear that this tensor field vanishes if and only if $M$ is Einstein. Q.E.D.

If we define a closed $2 k$-form $\gamma_{k}$ by

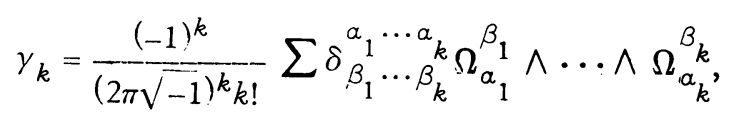

then the $k$ th Chern class $c_{k}$ of $M$ is represented by $\gamma_{k}$. In particular, $c_{1}$ and $c_{2}$ are represented by

$$
\gamma_{1}=\frac{\sqrt{-1}}{2 \pi} \sum \Omega_{\alpha}^{\alpha} \text { and } \gamma_{2}=-\frac{1}{8 \pi^{2}} \sum\left(\Omega_{\alpha}^{\alpha} \wedge \Omega_{\beta}^{\beta}-\Omega_{\beta}^{\alpha} \wedge \Omega_{a}^{\beta}\right)
$$

respectively.

3. Proof of Theorem. It follows from assumption (i) that

$$
\gamma_{1}^{n-2} \gamma_{2}=n \gamma_{1}^{n} / 2(n+1)+d \eta
$$

for some $(2 n-1)$-form $\eta$.

From assumption (ii), we have $S=\rho g / 2 n$ and, hence,

$$
\sum \Omega_{\alpha}^{\alpha}=\frac{\rho}{4 n} \sum \omega^{\alpha} \wedge \bar{\omega}^{\alpha}=\frac{\rho}{2 \sqrt{-1} n} \Phi
$$

This, together with (1) and (2), implies that

$$
\gamma_{1}=\frac{\rho}{4 n \pi} \Phi \quad \text { and } \quad \gamma_{2}=\frac{\rho^{2}}{32 n^{2} \pi^{2}} \Phi^{2}+\frac{1}{8 \pi^{2}} \sum \Omega_{\beta}^{a} \wedge \Omega_{\alpha}^{\beta}
$$

Therefore (3) is reduced to

$$
\frac{\rho^{n}}{2(n+1)(4 n \pi)^{n}} \Phi^{n}+\frac{2 n^{2} \rho^{n-2}}{(4 n \pi)^{n}} \Phi^{n-2} \wedge\left(\sum \Omega_{\beta}^{\alpha} \wedge \Omega_{\alpha}^{\beta}\right)=d \eta \text {. }
$$

Let $\Lambda$ be the operator of interior product by $\Phi$. Applying $\Lambda^{n}$ to both sides of (4), we may obtain

$$
\frac{n ! n ! \rho^{n}}{2(n+1)(4 n \pi)^{n}}+\frac{n^{2} n !(n-2) ! \rho^{n-2}}{(4 n \pi)^{n}}\left(1 / 2\|R\|^{2}-\|S\|^{2}\right)=\Lambda^{n} d \eta
$$

Integrating bot'l sides of this equation, we have

$$
\int_{M} \rho^{n-2}\left\{\frac{1}{2(n+1)} \rho^{2}+\frac{n}{2(n-1)}\|R\|^{2}-\frac{n}{n-1}\|S\|^{2}\right\} * 1=0
$$

where $* 1$ denotes the volume element of $M$. 
From assumption (ii) and Lemma, $\rho^{2}=2 n\|S\|^{2}$ and, hence, (5) can be written as

$$
\rho^{n-2} \int_{M}\left(\|R\|^{2}-\frac{4}{n+1}\|S\|^{2}\right) * 1=0
$$

This, together with Lemma, implies that either $\rho=0$ or $\|R\|^{2}=4\|S\|^{2} /(n+1)$. Therefore, $M$ is either Ricci-flat or of constant holomorphic sectional curvature.

\section{REFERENCES}

1. B.-Y. Chen and K. Ogiue, Some characterizations of complex space forms in terms of Chern classes, Quart. J. Math. Oxford Ser. 27 (1976).

2. S. I. Goldberg, Curvature and homology, Pure and Appl. Math., vol. 11, Academic Press, New York and London, 1962. MR 25\#2537.

DEPARTMENT OF MATHEMATICS, MICHIGAN STATE UNIVERSITY, EAST LANSING, MICHIGAN 48824

DEPARTMENT OF MATHEMATICS, TOKYO METROPOLITAN UNIVERSITY, SETAGAYA-KU, TOKYO, 158 JAPAN 